\title{
BMJ Open Social participation and risk of influenza infection in older adults: a cross-sectional study
}

\author{
Yugo Shobugawa, ${ }^{1}$ Takeo Fujiwara, ${ }^{2}$ Atsushi Tashiro, ${ }^{3}$ Reiko Saito, ${ }^{1}$ \\ Katsunori Kondo ${ }^{4,5}$
}

To cite: Shobugawa Y, Fujiwara T, Tashiro A, et al. Social participation and risk of influenza infection in older adults: a crosssectional study. BMJ Open 2018;8:e016876. doi:10.1136/ bmjopen-2017-016876

- Prepublication history for this paper is available online. To view these files please visit the journal online (http://dx.doi. org/10.1136/bmjopen-2017016876).

Received 16 March 2017 Revised 27 July 2017 Accepted 28 July 2017

Check for updates

${ }^{1}$ Division of International Health, Graduate School of Medical and Dental Sciences, Niigata University, Niigata, Japan ${ }^{2}$ Department of Global Health Promotion, Tokyo Medical and Dental University, Tokyo, Japan ${ }^{3}$ Niigata City Public Health Center, Niigata, Japan

${ }^{4}$ Center for Preventive Medical Sciences, Chiba University, Chiba, Japan

${ }^{5}$ Department of Gerontology and Evaluation Study, Center for Gerontology and Social Science, National Center for Geriatrics and Gerontology, Obu, Japan

Correspondence to

Dr Yugo Shobugawa;

yugo@med.niigata-u.ac.jp

\section{ABSTRACT}

Objectives Influenza infection can cause severe pneumonia, which is sometimes fatal, particularly in older adults. Influenza results in 3-5 million cases of severe illness and about 250000 to 500000 deaths annually worldwide. Social participation in the context of influenza infection is controversial because, although social participation is beneficial in maintaining physical function and mental health, it also increases the risk of contact with infected people. This study examined the association between social participation and influenza infection in Japanese adults aged 65 years or older.

Design Cross-sectional study.

Setting Japanese functionally independent adults aged 65 years or older.

Participants Among the respondents to the Japan Gerontological Evaluation Study (JAGES) 2013 survey, which took place during the period from 0ctober to December 2013, 12231 men and 14091 women responded to questions on influenza vaccination and influenza infection.

Outcome measures Using JAGES data for 12231 men and 14091 women aged $\geq 65$ years, we examined the association between social participation and influenza infection. The association between influenza infection and number of groups in which respondents participated was investigated among adults aged $\geq 65$ years, stratified by vaccination status and sex.

Results Unvaccinated women who participated in two or more social activities were 2.20 times $(95 \% \mathrm{Cl} 1.47$ to 3.29) as likely to report an influenza infection as those who reported no social participation. In contrast, vaccinated women who participated in two or more social groups had no additional risk of influenza infection as compared with female elders with no social participation. Among men, participation in social activities was not significantly associated with influenza infection, regardless of vaccination status. Conclusions Social participation was associated with a higher risk of influenza infection among unvaccinated older women, which suggests a need for further efforts to promote influenza vaccination, particularly among socially active elderly women.

\section{INTRODUCTION}

Influenza epidemics are estimated to result in 3-5 million cases of severe illness and about 250000 to 500000 deaths annually worldwide. ${ }^{1}$

\section{Strengths and limitations of this study}

- Although social participation is highly recommended for older adults, this study is the first to examine the association between social participation and influenza infection among this population.

- A strength of this study is that it used data from a large-scale social survey, which yielded information on influenza infection, influenza vaccination status and social participation.

- The study is based on cross-sectional data, which do not allow determination of causal relationships between influenza infection and social participation.

Older adults are disproportionally affected, ${ }^{2-4}$ as they are vulnerable to influenza infection and development of secondary bacterial pneumonia due to complications of chronic conditions such as congestive heart failure and chronic obstructive pulmonary disease. ${ }^{5}$ Influenza infection may also result in declines in critical physical functions in frail elders. ${ }^{6}$ Influenza infection is thus one of the most important causes of death in an ageing society.

Influenza infection is transmitted from person to person; therefore, social contact increases the risk of influenza infection..$^{7-10}$ However, social participation, which increases social contact, is beneficial for the physical and mental health of older adults. ${ }^{11-13}$ Previous research on social participation showed wide-ranging health benefits for older adults. Older adults who participate in social activities have better self-rated health, ${ }^{11}$ lower risks of disability, functional decline, mobility decline, depression, generalised anxiety disorders, cognitive decline and dementia, ${ }^{14-19}$ and longer lifespans. ${ }^{12}$ To our knowledge, no study has investigated the association between social participation and influenza infection among older adults.

Influenza vaccination status must be considered in any study of the association between influenza infection and social participation 
among adults aged $\geq 65$ years. Influenza vaccination is recommended for older adults, ${ }^{2021}$ as it decreases the risk of preventable death. ${ }^{1}$ For elders in Japan, vaccination fees are partly subsidised by the local government, to increase vaccination coverage. ${ }^{1415}$ The influenza vaccine must be altered to remain effective against currently circulating strains. The effectiveness of influenza vaccinations varies because the circulating type and subtype of the virus change every year. Thus, protection is sometimes greatly reduced or absent, especially in older adults. ${ }^{22}$ This study is one of the few to show the effectiveness of influenza vaccination in older adults. Because social participation is positively associated with influenza vaccination, ${ }^{23}$ the association between social participation and influenza infection might vary in relation to vaccination status. In addition, modes of contact during social participation might differ between men and women.

The Japan Gerontological Evaluation Study (JAGES) project is one of the largest cohort studies of social determinants of health among Japanese adults aged $\geq 65$ years. This study used part of the 2013 wave data from the 138294 respondents to the survey (response rate, $70.8 \%$ ). This study is the first to examine the association between social participation and influenza infection among elders stratified by influenza vaccination status and sex. In addition, we attempted to identify social activities that were associated with influenza infection.

\section{METHODS}

\section{Study population}

This study used data from the JAGES project, an ongoing prospective cohort study of the social determinants of health among functionally independent adults aged $\geq 65$ years. Several studies have used data from this large-scale nationwide project. ${ }^{24}{ }^{25}$ The cohort covers 30 municipalities in 13 prefectures in Japan. We used the 2013 wave of JAGES, in which questionnaires were mailed to a random sample of approximately 200000 community-dwelling individuals aged 65 years and older from October to December 2013. In addition to the basic items, five survey modules covered a variety of other topics. Module A covered nursing care, medical care and lifestyle; module $\mathrm{B}$ assessed oral hygiene, optimism and subjective health; module $\mathrm{C}$ covered social capital and history of abuse; module D evaluated subjective quality of life, sleep and influenza infection; and module $\mathrm{E}$ assessed physical activity. We used module $\mathrm{D}$, which included questions on influenza vaccination and influenza infection. All valid responses (from 12231 men and 14091 women) to module $\mathrm{D}$ were analysed.

\section{Influenza infection and vaccination}

Influenza infection status was determined by participant response to a self-administered questionnaire. To determine influenza infection status, respondents were asked, "Were you infected with influenza during the previous year? (yes, no)". Vaccination status was evaluated by asking respondents, "Did you receive an influenza vaccination during the previous year? (yes, no)".

\section{Social participation}

Social participation was defined as involvement in any social activity during the study period. ${ }^{26}$ Respondents were asked how often they took part in volunteer groups, sport groups or clubs, leisure activity groups, senior citizen clubs, neighbourhood associations, study or cultural groups, nursing care prevention for health promotion groups, in teaching skills or passing on experiences to others, local events, protection for older people, assistance for older people, child-rearing support, local environment improvement activities and other groups (frequency of participation: $\geq 4$ times per week, 2-3 times per week, once a week, 1-3 times per month, several times per year or never). We defined a participation frequency of at least 1-3 times per month as participation in a group, counted the number of groups in which the respondent participated and categorised participation as 0,1 or $\geq 2$ groups.

\section{Covariates}

Physical health status, particularly presence of respiratory disease, might be associated with social participation and influenza infection. ${ }^{27}{ }^{28}$ Self-rated health and respiratory disease as an underlying medical condition were assessed via questionnaire. Self-rated health was assessed by the question, "What is your current health status? (excellent, good, fair or poor)". Responses of excellent and good were classified as 'good' and responses of fair and poor as 'not good'. Presence of grandchildren in the household, which might be associated with social participation and influenza infection, was ascertained via questionnaire. Socioeconomic status was analysed as a possible confounder. Educational attainment was categorised as $<6$ years, $6-9$ years, $10-12$ years, $\geq 13$ years and other. Household income was equalised by the square root of the number of household members and classified as $<1.99$ million yen, 2-3.99 million yen and $>4$ million yen. Age was categorised into five groups: $65-69,70-74$, $75-79,80-84$ and $\geq 85$ years.

\section{Analysis}

Logistic regression analysis was used to examine the association between social participation and influenza infection. When an interaction term between a factor and social participation was significantly associated with influenza infection, analyses were done after stratifying respondents by that factor. We adjusted for the following possible confounding factors: age, self-rated health, underlying respiratory disease, living with grandchildren, educational attainment and equivalent income.

\section{Additional analysis}

To determine whether certain types of social activity were more likely to result in influenza infection, we set participation in each activity as an explanatory variable, instead of participation in any social activity, and this 
yielded 14 models. Data from all the respondents were analysed. Number of participating activities was included as a covariate, to measure the effect of participating in each activity. ORs for influenza infection were calculated in relation to participation in each activity, stratified by vaccination status and sex. All analyses were performed with STATA SE V.13 (StataCorp).

\section{Ethical considerations}

The questionnaire and an explanation of the study were sent to participants via mail. The recipients were informed that participation was voluntary and that returning the self-administered questionnaire would be interpreted as consent to participate. Ethical approval for the study was obtained from the ethics committee at Nihon Fukushi University.

\section{RESULTS}

The interaction term between vaccination status and social participation was significantly associated with influenza infection, as was the interaction term between sex and social participation. Thus, all analyses of the groups were stratified by vaccination status and sex. The characteristics of the study sample, stratified by vaccination status and sex, are shown in table 1 . The vaccinated group was older than the unvaccinated group, and this was true for men and women. In particular, $49.5 \%$ of vaccinated men and $32.9 \%$ of unvaccinated men were 75 years or older; the respective values were $48.0 \%$ and $34.7 \%$ for women. Approximately one-third of vaccinated men $(29.1 \%)$ and vaccinated $(33.3 \%)$ women participated in two or more groups, while less than one-fourth $(22.6 \%)$ of unvaccinated men participated in two or more groups.

A total of 355 cases $(5.5 \%)$ and 372 cases $(4.2 \%)$ of influenza infection were observed among vaccinated men and women, respectively, and 136 cases (2.4\%) and 124 cases $(2.3 \%)$ of influenza infection were observed in unvaccinated men and women, respectively. The proportion of respondents with respiratory diseases was higher among vaccinated elders $(7.6 \%$ in men and $5.0 \%$ in women, $\mathrm{P}<0.001)$ than among unvaccinated elders $(4.0 \%$ in men and $3.4 \%$ in women, $\mathrm{P}<0.001)$. Vaccinated elders were more likely than unvaccinated elders to live with grandchildren (men: $16.2 \%$ vs $11.2 \%, \mathrm{P}<0.001$; women: $21.1 \%$ vs $13.3 \%, \mathrm{P}<0.001)$.

Table 2 shows the results of logistic analysis of influenza infection in respondents stratified by influenza vaccination status and sex. Overall, unvaccinated women who participated in two or more social activities were more likely to develop influenza than were unvaccinated women who did not participate in such activities (OR 2.20; 95\% CI 1.47 to 3.29), after adjustment for age, self-rated health, presence of respiratory disease, living with grandchildren and socioeconomic status (educational attainment and equivalent income). However, among vaccinated women, social participation in two or more activities was not associated with influenza infection after adjustment (OR 1.06; 95\% CI 0.83 to 1.36 ). Social participation was not associated with influenza infection among vaccinated or unvaccinated men. Participation in one group was not associated with influenza infection in either group. Presence of respiratory disease was significantly associated with influenza infection among vaccinated men (OR 1.94; 95\% CI 1.40 to 2.71), unvaccinated men (OR 2.44; $95 \%$ CI 1.28 to 4.68 ) and vaccinated women (OR 1.89; $95 \%$ CI 1.31 to 2.73). Poor self-rated health was significantly associated with influenza infection in vaccinated (OR 1.49; $95 \%$ CI 1.15 to 1.92 ) and unvaccinated women (OR $1.80 ; 95 \%$ CI 1.12 to 2.87 ).

Each social participation was not associated with influenza infection among vaccinated elderly men or women (figures 1 and 2). Among unvaccinated elders (figures 3 and 4), only men who participated in a leisure activity group had a significantly decreased risk of influenza infection (OR $0.56 ; 95 \% \mathrm{CI}-0.33$ to 0.94 ; figure 3 ).

\section{DISCUSSION}

In this study, risk of influenza infection was higher for unvaccinated elders, particularly women, than for vaccinated Japanese elders. This suggests that influenza vaccination is effective in preventing influenza infection among active older adults and highlights the urgent need for additional efforts to promote influenza vaccination among socially active elders, especially women.

In an analysis of upper respiratory tract viruses, such as rhinovirus, in a quarantine setting, Cohen et al found that for unknown reasons individuals with diverse social networks had greater resistance to upper respiratory illnesses. ${ }^{29}$ It is hypothesised that certain immune mechanism operates as pathways and that behaviour affects release of cytokines in nasal passages. Similarly, establishing a social network through group activities might prevent viral infection. However, frequent contact with infected persons could result in infection even among people with robust social networks. In our study, the risk of influenza infection was not higher among vaccinated elders when the likelihood of contact increased. When available, vaccines might help prevent transmission of infectious respiratory agents other than influenza virus, as is the case for the pneumococcal vaccine. ${ }^{30}$ However, when no vaccine is available, non-pharmaceutical interventions such as respiratory hygiene and cough etiquette ${ }^{3132}$ may be effective preventive measures. Future studies should investigate infections other than influenza.

In this study, participation in social activities did not increase the risk of influenza infection among vaccinated elders, even after adjustment for confounding factors. In general, influenza vaccines provide protection against influenza infection. ${ }^{21}{ }^{33}$ Several previous studies reported that vaccination reduced hospitalisations and deaths in older adults. ${ }^{34-36}$ Our results 


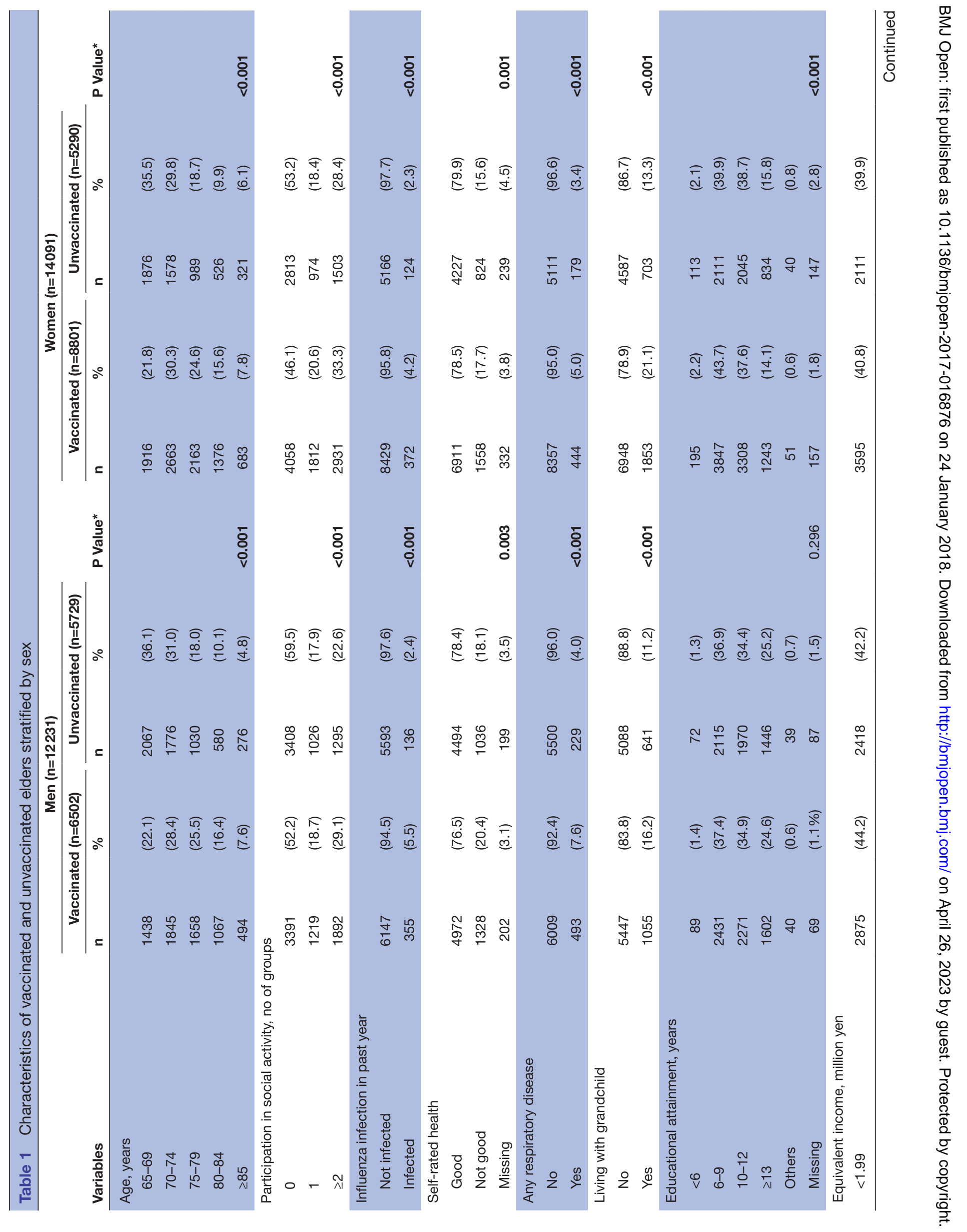




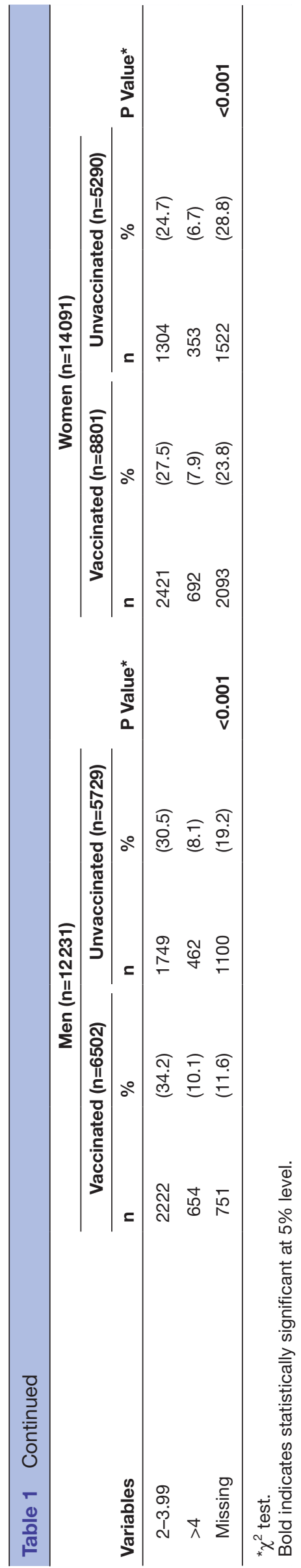

confirm the effectiveness of influenza vaccines, but only in women. Protection is sometimes greatly reduced or absent, especially in older adults. ${ }^{22}$ This study is one of the few that have confirmed the effectiveness of influenza vaccination in older adults.

Respondents were stratified by vaccination status because it may modify the association between social participation and influenza infection. Older people who participate in two or more groups are more likely to be vaccinated than are those who do not participate in such activities. It is believed that elders who participate in social activities have good access to health information, including how and where they can receive influenza vaccinations. ${ }^{20} 3738$ Gathering for any group activity means sharing information in participant interests, such as health information, even when the aim of the activity may not be directly related to such interests. In addition, socially active persons have better medical access, which includes vaccinations and consultation with physicians. ${ }^{20}$ This suggests that social persons who participate in groups are more likely than non-social persons to see a doctor when they develop a fever, especially in Japan, where medical resources are accessible to the entire population.

We also stratified respondents by sex. Evidence indicates that antibody responses after vaccination are stronger for women than for men. ${ }^{39}$ Such differences in response were observed for various vaccines, including influenza vaccine. ${ }^{4041}$ This could explain the sex differences observed in this study. Behavioural differences between men and women may also have a role. Women tend to talk more than men during social activities. The risk of influenza infection was higher in unvaccinated women who participated in two or more groups.

The risk of influenza infection varied in relation to the type of social activity and by sex, but most activities did not significantly increase infection risk in vaccinated or unvaccinated elders. Only participation in a leisure activity group was associated with decreased risk for infection, among unvaccinated men, perhaps because such groups have less opportunities for close contact. ${ }^{23}$ However, reason why a significant association was observed only in unvaccinated men was unclear. One reason for the lack of a significant association with most activities was larger effect of number of participation rather than type of social activity. Transmission of the influenza virus between humans is mainly by respiratory droplets, although airborne transmission is possible. ${ }^{42} 43$ Thus, activities with fewer opportunities for conversation and direct contact have less infection risk. Similarly, the risk of transmission is higher for larger groups. ${ }^{43}{ }^{44}$ In our study, group size and number of people gathered were unknown. Future studies should investigate why infection risk varies by group type and sex.

Our study has several limitations. First, because it is cross-sectional, causality cannot be inferred. A longitudinal study or a randomised controlled trial is 


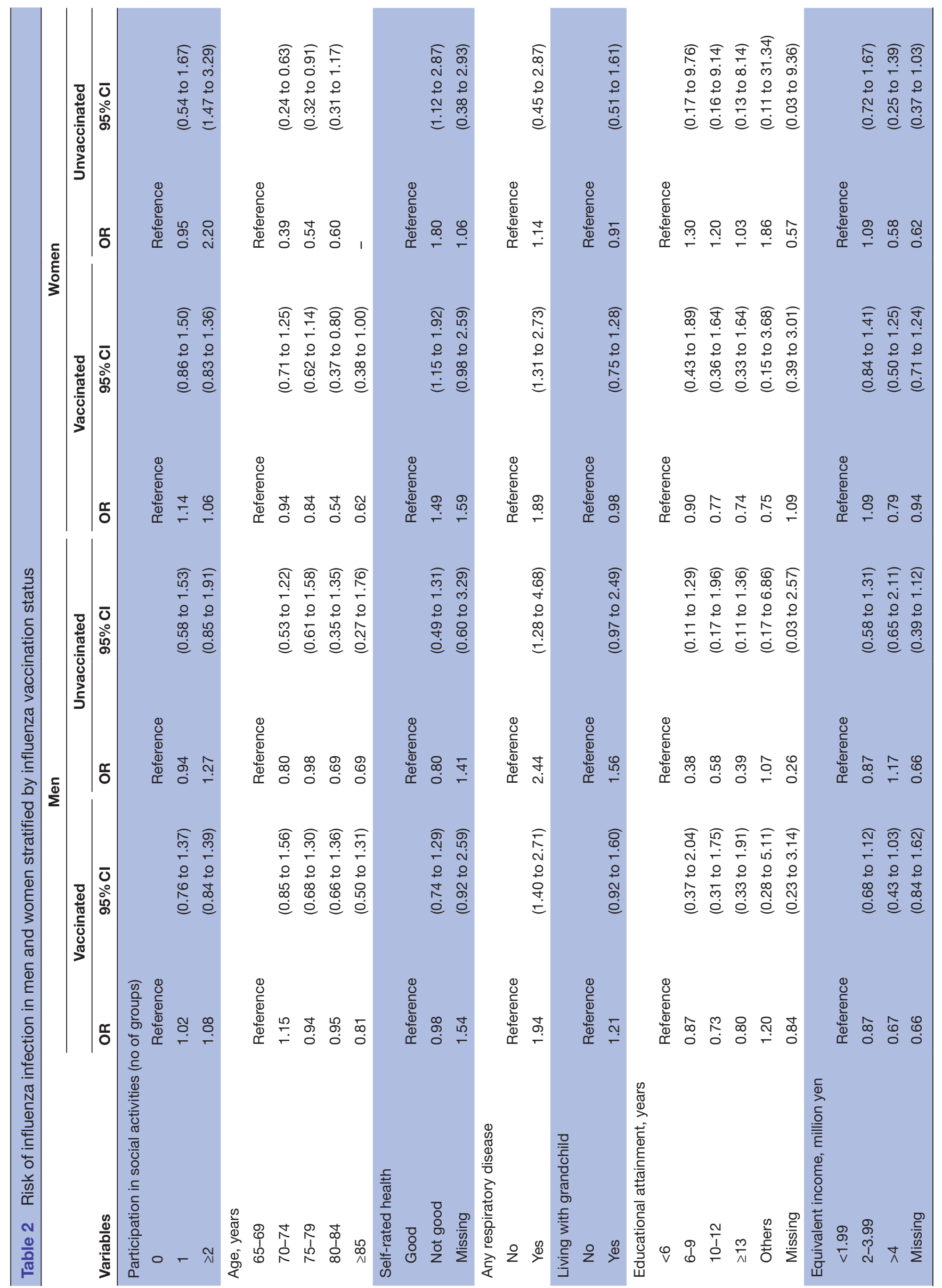


Vaccinated men $(\mathrm{N}=6,502)$

\begin{tabular}{lccc} 
Social activity & OR & $95 \% \mathrm{Cl}$ \\
\hline Volunteer group & 0.89 & $(0.58-1.36)$ \\
Sport group or club & 1.03 & $(0.74-1.42)$ \\
Leisure activity group & 0.82 & $(0.59-1.14)$ \\
Senior citizen club & 1.43 & $(0.96-2.12)$ \\
Neighbourhood association & 1.44 & $(0.98-2.10)$ \\
Study or cultural group & 1.10 & $(0.67-1.82)$ \\
Nursing care prevention for health-building & 0.78 & $(0.44-1.36)$ \\
Teaching skills or passing on experiences to others & 1.19 & $(0.71-1.98)$ \\
Local events & 0.78 & $(0.36-1.71)$ \\
Protection for older people & 0.63 & $(0.30-1.33)$ \\
Assistance for older people & 0.60 & $(0.26-1.36)$ \\
Child-rearing support & 0.85 & $(0.44-1.65)$ \\
Local environment improvement & 0.85 & $(0.49-1.49)$ \\
Other & 1.04 & $(0.65-1.68)$ \\
\hline
\end{tabular}

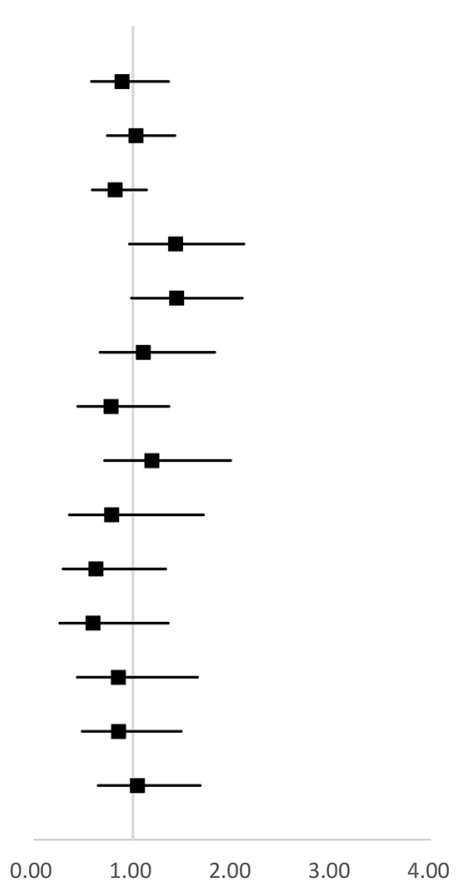

Odds ratio

Figure 1 ORs for influenza infection in relation to participation in specific social activities among vaccinated men. Logistic regression was adjusted for age, number of participating groups, self-rated health, respiratory disease, living with grandchildren and socioeconomic status.

\section{Vaccinated women $(\mathrm{N}=8,801)$}

\begin{tabular}{lccc} 
Social activity & OR & $95 \% \mathrm{Cl}$ \\
\hline Volunteer group & 0.82 & $(0.53-1.29)$ \\
Sport group or club & 1.03 & $(0.75-1.41)$ \\
Leisure activity group & 1.04 & $(0.76-1.40)$ \\
Senior citizen club & 0.99 & $(0.66-1.48)$ \\
Neighbourhood association & 1.15 & $(0.73-1.82)$ \\
Study or cultural group & 0.85 & $(0.55-1.31)$ \\
Nursing care prevention for health-building & 1.30 & $(0.87-1.96)$ \\
Teaching skills or passing on experiences to others & 0.69 & $(0.37-1.30)$ \\
Local events & 1.38 & $(0.60-3.19)$ \\
Protection for older people & 0.52 & $(0.25-1.08)$ \\
Assistance for older people & 0.97 & $(0.52-1.81)$ \\
Child-rearing support & 1.13 & $(0.61-2.10)$ \\
Local environment improvement & 1.23 & $(0.71-2.14)$ \\
Other & 1.32 & $(0.80-2.18)$ \\
\hline
\end{tabular}

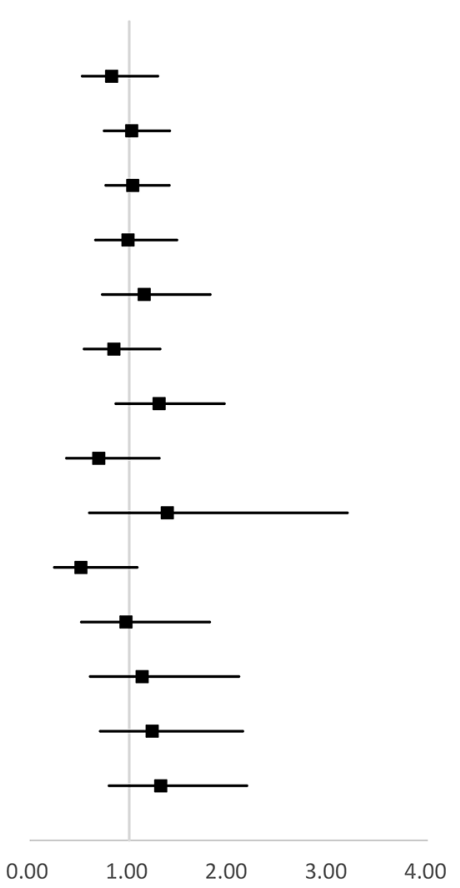

Odds ratio

Figure 2 ORs for influenza infection in relation to participation in specific social activities among vaccinated women. Logistic regression was adjusted for possible confounders. 
Unvaccinated men $(\mathrm{N}=5,729)$

\begin{tabular}{lcc} 
Social activity & OR & $95 \% \mathrm{Cl}$ \\
\hline Volunteer group & 1.16 & $(0.59-2.26)$ \\
Sport group or club & 1.55 & $(0.95-2.54)$ \\
Leisure activity group & 0.56 & $(0.33-0.94)$ \\
Senior citizen club & 1.10 & $(0.54-2.24)$ \\
Neighbourhood association & 0.71 & $(0.36-1.40)$ \\
Study or cultural group & 0.99 & $(0.45-2.20)$ \\
Nursing care prevention for health-building & 1.04 & $(0.43-2.51)$ \\
Teaching skills or passing on experiences to others & 1.14 & $(0.54-2.43)$ \\
Local events & 1.13 & $(0.42-3.08)$ \\
Protection for older people & 1.18 & $(0.42-3.28)$ \\
Assistance for older people & 1.52 & $(0.55-4.20)$ \\
Child-rearing support & 0.82 & $(0.29-2.31)$ \\
Local environment improvement & 0.95 & $(0.43-2.10)$ \\
Other & 0.83 & $(0.40-1.71)$ \\
\hline
\end{tabular}

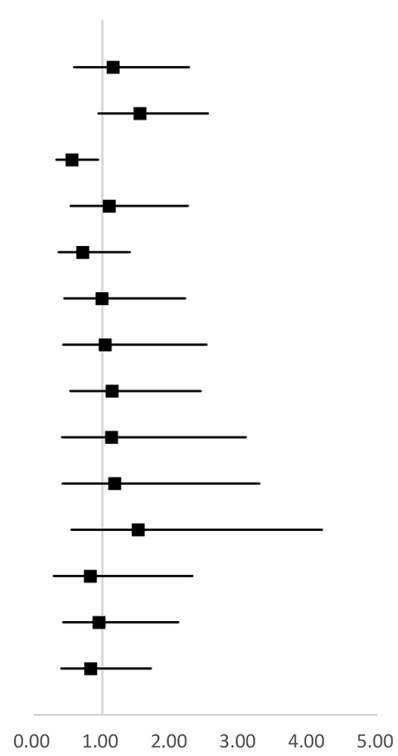

Odds ratio

Figure 3 ORs for influenza infection in relation to participation in specific social activities among unvaccinated men. Logistic regression was adjusted for possible confounders.

\begin{tabular}{lcc} 
Unvaccinated women $(\mathrm{N}=5,290)$ & & \\
Social activity & OR & $95 \% \mathrm{Cl}$ \\
\hline Volunteer group & 0.65 & $(0.31-1.36)$ \\
Sport group or club & 0.93 & $(0.55-1.55)$ \\
Leisure activity group & 1.11 & $(0.69-1.81)$ \\
Senior citizen club & 1.74 & $(0.91-3.31)$ \\
Neighbourhood association & 1.07 & $(0.54-2.14)$ \\
Study or cultural group & 0.78 & $(0.39-1.54)$ \\
Nursing care prevention for health-building & 1.19 & $(0.60-2.36)$ \\
Teaching skills or passing on experiences to others & 0.44 & $(0.18-1.09)$ \\
Local events & 0.69 & $(0.19-2.47)$ \\
Protection for older people & 1.13 & $(0.48-2.62)$ \\
Assistance for older people & 1.53 & $(0.69-3.39)$ \\
Child-rearing support & 0.64 & $(0.24-1.69)$ \\
Local environment improvement & 0.84 & $(0.34-2.07)$ \\
Other & 1.23 & $(0.61-2.46)$ \\
\hline
\end{tabular}

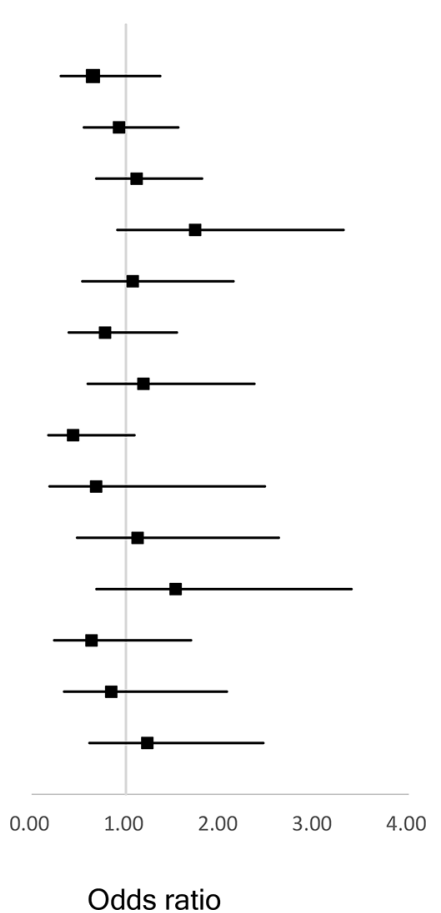

Figure 4 ORs for influenza infection in relation to participation in specific social activities among unvaccinated women. Logistic regression was adjusted for possible confounders.

needed in order to prove a causal relationship between social participation and influenza infection. Second, diagnosis of influenza infection was based on self-reports rather than on the results of laboratory testing. However, in Japan, commercial rapid diagnostic test kits are commonly used in clinical settings such as outpatient clinics. These kits have high sensitivity and specificity in the diagnosis of influenza infection. ${ }^{45} 46$ In addition, medical access is good because of the universal health insurance system in Japan. ${ }^{47}$ Older adults with an influenza-like illness in Japan are generally tested with rapid diagnostic tests. ${ }^{45} 48$ Third, vaccination history was self-reported. Fourth, previous influenza infection was not assessed. 
In conclusion, social participation increased the risk of influenza infection among unvaccinated elders, particularly women, which suggests that additional efforts are needed in order to encourage influenza vaccination among socially active elders.

Acknowledgements This research project is associated with and used data from the Japan Gerontological Evaluation Study (JAGES), which was conducted by the Center for Well-being and Society, Nihon Fukushi University. We thank Ms Tomoko Manabe for her excellent secretarial work. We are extremely grateful to the study participants for the use of their personal data and would like to express our deepest gratitude to the members of the JAGES Project for their painstaking efforts in the conduct of the survey.

Collaborators The members of the JAGES group are as follows: Kondo $\mathrm{K}$ (the lead investigator), Hanazato M, Hikichi H, Miyaguni Y, Sasaki Y, Nagamine Y, Chiba University, Chiba; Ashida T, Kondo N, Takagi D, Tani Y, The University of Tokyo, Tokyo; Aida J, Osaka K, Tsuboya T, Tohoku University, Miyagi; Jeong S, Murata C, Saito T, National Center for Geriatrics and Gerontology, Aichi; Ojima T, Okada E, Hamamatsu University School of Medicine, Shizuoka; Shirai K, Todoriki H, University of the Ryukyus, Okinawa; Saito M, Nihon Fukushi University, Aichi; Hirai H, Iwate University, Iwate; Misawa J, Rikkyo University, Tokyo; Suzuki K, Aichi Gakuin University, Aichi; Ichida Y, Doctoral Institute for Evidence Based Policy, Tokyo; Takeda T, Seijoh University, Aichi; Yamamoto T, Kanagawa Dental University, Kanagawa; Nakade M, Tokaigakuen University, Aichi; Cable N, University College London, London; Tamakoshi A, Hokkaido University Graduate School of Medicine, Hokkaido; Fujino Y, University of Occupational and Environmental Health, Fukuoka; Hayashi T, Tokai College of Medical Science, Aichi.

Contributors All authors met the International Committee of Medical Journal Editors criteria for authorship. YS and TF contributed to the study design. YS performed statistical analysis and drafted the manuscript. TF advised on data analysis and interpretation. RS, KK and TF revised the manuscript. KK is the principal investigator of the JAGES project. AT helped to develop the idea of the study. All authors read and approved the final manuscript.

Funding This work was supported by JSPS KAKENHI Grant Number 26460828. This study was also supported by a Health Labour Sciences Research Grant for conduct of a nationwide survey. Additional support included grants for Comprehensive Research on Aging and Health (H26-Choju-Ippan-006, H25-Choju-Ippan-003, H25-Kenki-Wakate-015, H25-Irryo-Shitei-003 [Fukkou], H24-Junkanki [Syosyu]-Ippan-007) from the Japanese Ministry of Health, Labour and Welfare, grants from the Department of Health and Human Services, the National Institutes of Health, Grants-in-Aid for Scientific Research (20319338, 22390400, 23243070, 23590786, 23790710, 24140701, 24390469, 24530698, $24653150,24683018,25253052,25870881$ ) from the Japan Society for the Promotion of Science, and a grant from the National Center for Geriatrics and Gerontology (number: 24-17, Chiyoe Murata; number: 24-23, Tami Saito; number: J09KF00804, Yuri Sasaki).

Competing interests None declared.

Patient consent Obtained.

Ethics approval Nihon Fukushi University.

Provenance and peer review Not commissioned; externally peer reviewed.

Data sharing statement No additional data are available.

Open Access This is an Open Access article distributed in accordance with the Creative Commons Attribution Non Commercial (CC BY-NC 4.0) license, which permits others to distribute, remix, adapt, build upon this work non-commercially, and license their derivative works on different terms, provided the original work is properly cited and the use is non-commercial. See: http://creativecommons.org/ licenses/by-nc/4.0/

(c) Article author(s) (or their employer(s) unless otherwise stated in the text of the article) 2018. All rights reserved. No commercial use is permitted unless otherwise expressly granted.

\section{REFERENCES}

1. World Health Organization. Influenza (Seasonal) Fact sheet $N^{\circ} 211$ 2014 [updated March 2014. http://www.who.int/mediacentre/ factsheets/fs211/en/ (accessed 19 Aug 2015).
2. Thompson WW, Moore MR, Weintraub E, et al. Estimating influenzaassociated deaths in the United States. Am J Public Health 2009;99(Suppl 2):S225-S230.

3. Thompson WW, Weintraub E, Dhankhar P, et al. Estimates of US influenza-associated deaths made using four different methods. Influenza Other Respir Viruses 2009;3:37-49.

4. Centers for Disease Control and Prevention (CDC). Estimates of deaths associated with seasonal influenza --- United States, 19762007. MMWR Morb Mortal Wkly Rep 2010;59:1057-62.

5. Schanzer DL, Langley JM, Tam TW. Co-morbidities associated with influenza-attributed mortality, 1994-2000, Canada. Vaccine 2008;26:4697-703.

6. Barker WH, Borisute $\mathrm{H}$, Cox C. A study of the impact of influenza on the functional status of frail older people. Arch Intern Med 1998;158:645-50.

7. Killingley B, Nguyen-Van-Tam J. Routes of influenza transmission. Influenza Other Respir Viruses 2013;7 Suppl 2:42-51.

8. Jones RM. Critical review and uncertainty analysis of factors influencing influenza transmission. Risk Anal 2011;31:1226-42.

9. Smieszek T, Fiebig L, Scholz RW. Models of epidemics: when contact repetition and clustering should be included. Theor Biol Med Model 2009;6:11

10. Eames KT. Modelling disease spread through random and regular contacts in clustered populations. Theor Popul Biol 2008;73:104-11.

11. Ichida $\mathrm{Y}$, Hirai $\mathrm{H}$, Kondo $\mathrm{K}$, et al. Does social participation improve self-rated health in the older population? A quasi-experimental intervention study. Soc Sci Med 2013;94:83-90.

12. Aijö M, Parkatti T. Independent and combined association of physical activity and cardiac disease on mortality risk in the very old. $J$ Aging Health 2011;23:70-85.

13. Hyyppä MT, Mäki J. Social participation and health in a community rich in stock of social capital. Health Educ Res 2003;18:770-9.

14. Avlund $\mathrm{K}$, Vass M, Hendriksen $\mathrm{C}$. Onset of mobility disability among community-dwelling old men and women. The role of tiredness in daily activities. Age Ageing 2003;32:579-84.

15. Buchman AS, Boyle PA, Wilson RS, et al. Association between latelife social activity and motor decline in older adults. Arch Intern Med 2009;169:1139-46

16. Mendes de Leon CF, Glass TA, Berkman LF. Social engagement and disability in a community population of older adults: the New Haven EPESE. Am J Epidemiol 2003;157:633-42.

17. James BD, Boyle PA, Buchman AS, et al. Relation of late-life social activity with incident disability among community-dwelling older adults. J Gerontol A Biol Sci Med Sci 2011;66:467-73.

18. Thomas PA. Trajectories of social engagement and limitations in late life. J Health Soc Behav 2011;52:430-43.

19. Kanamori S, Kai Y, Aida J, et al. Social participation and the prevention of functional disability in older Japanese: the JAGES cohort study. PLoS One 2014;9:e99638.

20. Crawford VL, O'Hanlon A, McGee H. The effect of patient characteristics upon uptake of the influenza vaccination: a study comparing community-based older adults in two healthcare systems. Age Ageing 2011;40:35-41.

21. Grohskopf LA, Sokolow LZ, Olsen SJ, et al. Prevention and Contro of Influenza with Vaccines: Recommendations of the Advisory Committee on Immunization Practices, United States, 2015-16 Influenza Season. MMWR Morb Mortal Wkly Rep 2015;64:818-25.

22. Jefferson T, Rivetti D, Rivetti A, et al. Efficacy and effectiveness of influenza vaccines in elderly people: a systematic review. Lancet 2005;366:1165-74

23. Ibuka $Y$, Ohkusa $Y$, Sugawara $T$, et al. Social contacts, vaccination decisions and influenza in Japan. $J$ Epidemiol Community Health 2016;70.

24. Hayashi K, Kawachi I, Ohira T, et al. Laughter is the Best Medicine? A Cross-Sectional Study of Cardiovascular Disease Among Older Japanese Adults. Journal of epidemiology / Japan Epidemiological Association 2016 (published Online First: 2016/03/15).

25. Yamakita M, Kanamori S, Kondo N, et al. Correlates of Regular Participation in Sports Groups among Japanese Older Adults: JAGES Cross-Sectional Study. PLoS One 2015;10:e0141638.

26. Floud S, Balkwill A, Canoy D, et al. Social participation and coronary heart disease risk in a large prospective study of UK women. Eur $J$ Prev Cardiol 2016;23.

27. Siu E, Campitelli MA, Kwong JC. Physical activity and influenzacoded outpatient visits, a population-based cohort study. PLoS One 2012;7:e39518. 
28. Choi SM, Jeong YJ, Park JS, et al. The impact of lifestyle behaviors on the acquisition of pandemic $(\mathrm{H} 1 \mathrm{~N} 1)$ influenza infection: a casecontrol study. Yonsei Med J 2014;55:422-7.

29. Cohen S, Doyle WJ, Skoner DP, et al. Social ties and susceptibility to the common cold. JAMA 1997;277:1940-4.

30. Pilishvili T, Bennett NM. Pneumococcal disease prevention among adults: Strategies for the use of pneumococcal vaccines. Vaccine 2015;33 Suppl 4:D60-D65.

31. Bell D, Nicoll A, Fukuda K, et al. Non-pharmaceutical interventions for pandemic influenza, national and community measures. Emerg Infect Dis 2006;12:88-94.

32. Aiello AE, Coulborn RM, Aragon TJ, et al. Research findings from nonpharmaceutical intervention studies for pandemic influenza and current gaps in the research. Am J Infect Control 2010;38:251-8.

33. Jefferson T, Di Pietrantonj C, Rivetti A, et al. Vaccines for preventing influenza in healthy adults. Cochrane Database Syst Rev 2014;3:CD001269.

34. Chan TC, Fan-Ngai Hung I, Ka-Hay Luk J, et al. Effectiveness of influenza vaccination in institutionalized older adults: a systematic review. J Am Med Dir Assoc 2014;15:226.e1-226.e6.

35. Christenson B, Lundbergh $\mathrm{P}$, Hedlund $\mathrm{J}$, et al. Effects of a large-scale intervention with influenza and 23-valent pneumococcal vaccines in adults aged 65 years or older: a prospective study. Lancet 2001;357:1008-11.

36. Nordin J, Mullooly J, Poblete S, et al. Influenza vaccine effectiveness in preventing hospitalizations and deaths in persons 65 years or older in Minnesota, New York, and Oregon: data from 3 health plans. $J$ Infect Dis 2001;184:665-70.

37. Victor JF, Gomes GD, Sarmento LR, et al. [Factors associated with vaccination against influenza $A(\mathrm{H} 1 \mathrm{~N} 1)$ in the elderly]. Rev Esc Enferm USP 2014;48:57-64
38. Francisco PM, Donalisio MR, Barros MB, et al. [Factors associated with vaccination against influenza in the elderly]. Rev Panam Salud Publica 2006;19:259-64.

39. Klein SL, Marriott I, Fish EN. Sex-based differences in immune function and responses to vaccination. Trans $R$ Soc Trop Med Hyg 2015;109:9-15.

40. Klein SL, Pekosz A. Sex-based biology and the rational design of influenza vaccination strategies. J Infect Dis 2014;209 (Suppl 3):S114-S119.

41. Fink AL, Klein SL. Sex and Gender Impact Immune Responses to Vaccines Among the Elderly. Physiology 2015;30:408-16.

42. Weber TP, Stilianakis NI. Inactivation of influenza A viruses in the environment and modes of transmission: a critical review. J Infect 2008;57:361-73.

43. Brankston G, Gitterman L, Hirji Z, et al. Transmission of influenza A in human beings. Lancet Infect Dis 2007;7:257-65.

44. Killingley B, Enstone J, Booy R, et al. Potential role of human challenge studies for investigation of influenza transmission. Lancet Infect Dis 2011;11:879-86.

45. Beckmann $\mathrm{C}$, Hirsch $\mathrm{HH}$. Diagnostic performance of near-patient testing for influenza. J Clin Virol 2015;67:43-6.

46. Cho $\mathrm{CH}$, Woo MK, Kim JY, et al. Evaluation of five rapid diagnostic kits for influenza A/B virus. J Virol Methods 2013;187:51-6.

47. Ikegami N, Yoo BK, Hashimoto $\mathrm{H}$, et al. Japanese universal health coverage: evolution, achievements, and challenges. Lancet 2011;378:1106-15.

48. Sugaya N. Widespread use of neuraminidase inhibitors in Japan. $J$ Infect Chemother 2011;17:595-601. 